\title{
CRÓNICA
}

\section{Creación musical chilena}

Cuadro sinóptico de obras de compositores chilenos interpretadas durante el segundo semestre (abril-septiembre 2016)

preparado por Nancy Sattler Jiménez

En cada entrada se indica el nombre del autor (a), seguido de la información relativa al título y medio para el que está escrita la obra (abreviado como TM), la fecha de presentación (abreviada como $F$ ), la ocasión (cuando corresponda) y el lugar de interpretación de la música (abreviados como $O L$ ), la individualización de los intérpretes (abreviado como Int) y cuando sea pertinente se indican los solistas (abreviados como Sol/Sols). Si una obra se repite más de una vez, no se reitera el título, sino que la secuencia de información se señala desde la fecha de presentación en adelante. En orden alfabético las abreviaturas son las siguientes.

$\begin{array}{ll}\text { arr:: } & \text { Arreglo } \\ \text { F: } & \text { Fecha } \\ \text { Int: } & \text { Intérprete, intérpretes } \\ \text { OL: } & \text { Ocasión y lugar } \\ \text { Sol, Sols: } & \text { Solista, Solistas } \\ \text { TM: } & \text { Título y medio }\end{array}$

Acevedo Elgueta, Claudio. TM: Hombre pájaro (2012) para conjunto instrumental; F: 26 de abril de 2016; OL: Concierto gratuito, Librería Le Monde Diplomatique, Santiago; Int: Grupo Sexto Piso, Claudio Acevedo (director).

TM: Danzas del sur del mundo (1. Gauchada, 2. Milongueña, 3. Cueca guaranga) (2002) para orquesta de flautas; F: 17 de mayo de 2016; OL: Temporada Oficial del Departamento de Música y Sonología, Facultad de Artes de la Universidad de Chile, Centro Cultural Gabriela Mistral (GAM). F: 25 de mayo de 2016. 22 de agosto de 2016; OL: Ciclo Música Chilena y Latinoamericana, Departamento de Música y Sonología, Facultad de Artes de la Universidad de Chile, Biblioteca Nacional. F: 23 de agosto de 2016; OL: Concierto Didáctico, Departamento de Música y Sonología, Facultad de Artes de la Universidad de Chile, Centro Cultural Gabriela Mistral (GAM); Int: Orquesta de Flautas Illawara, Wilson Padilla Véliz (director).

TM: Merengue (2012) para flautas; F: 22 de agosto de 2016; OL: Ciclo Música Chilena y Latinoamericana, Departamento de Música y Sonología, Facultad 
de Artes de la Universidad de Chile, Biblioteca Nacional; Int: Orquesta de Flautas Illawara, Wilson Padilla Véliz (director).

Acevedo Elgueta, Claudio / Josefina Echenique. TM: Luz y penumbra (2001) para conjunto vocal e instrumental; F: 26 de abril de 2016; OL: Concierto gratuito, Librería Le Monde Diplomatique, Santiago; Int: Grupo Sexto Piso, Claudio Acevedo (director).

Acevedo Elgueta, Claudio / Nicolás Guillén. TM: Canto negro (2013) para conjunto vocal e instrumental; F: 26 de abril de 2016; OL: Concierto gratuito, Librería Le Monde Diplomatique, Santiago; Int: Grupo Sexto Piso, Claudio Acevedo (director).

Acevedo Elgueta, Claudio / Carlos Pezoa Véliz. TM: Nada (2007) para conjunto vocal e instrumental; F: 26 de abril de 2016; OL: Concierto gratuito, Librería Le Monde Diplomatique, Santiago; Int: Grupo Sexto Piso, Claudio Acevedo (director).

Acevedo Elgueta, Claudio / Nicomedes Santa Cruz. TM: Como has cambiado pelona (2012) para conjunto vocal e instrumental; F: 26 de abril de 2016; OL: Concierto gratuito, Librería Le Monde Diplomatique, Santiago; Int: Grupo Sexto Piso, Claudio Acevedo (director).

Acosta, Víctor (arr. Guillermo Rifo). TM: La joya del pacífico (1942), versión para orquesta; F: 9, 10, 15 y 16 de septiembre de 2016; OL: Concierto $\mathrm{N}^{\circ} 17$, Música Chilena, Temporada Oficial 2016 de la Orquesta Sinfónica de Chile (OSCH), Centro de Extensión Artística y Cultural de la Universidad de Chile, Teatro Universidad de Chile; Int: Orquesta Sinfónica de Chile, Guillermo Rifo (director).

Advis Vitaglic, Luis. TM: Cantata Santa María de Iquique (1969) para conjunto vocal e instrumental; F: 21 de abril de 2016; OL: Celebración de los 50 años del conjunto Quilapayún, Teatro Municipal de Santiago; Int: Conjunto Quilapayún, junto a la Orquesta Sinfónica Estudiantil Metropolitana, Fernando Saavedra (director).

TM: Cueca (1978) para soprano y piano; F: 16 de junio de 2016; OL: Concierto de canto y piano, Academia Chilena de Bellas Artes, Salón de Honor del Instituto de Chile; Int: Camila García (soprano), Jacqueline Urízar (piano).

TM: Rin (1981) para soprano y piano; F: 16 de junio de 2016; OL: Concierto de canto y piano, Academia Chilena de Bellas Artes, Salón de Honor del Instituto de Chile; Int: Camila García (soprano), Jacqueline Urízar (piano).

Aguirre, Elvira. TM: Admiro tu belleza (siglo XIX) para piano; F: 28 de septiembre de 2016; OL: Temporada Oficial, Departamento de Música y Sonología, Facultad de Artes de la Universidad de Chile, Ciclo de piano, Sala Isidora Zegers; Int: Kenya Godoy (piano).

Alarcón Soto, Rolando (arr. Guillermo Rifo). TM: Si somos americanos (1969), versión para orquesta; F: 9, 10, 15 y 16 de septiembre de 2016; OL: Concierto $\mathrm{N}^{\mathrm{o}} 17$, Música Chilena, Temporada Oficial 2016, Celebración Fiestas Patrias, Centro de Extensión Artística y Cultural de la Universidad de Chile, Teatro Universidad de Chile; Int: Orquesta Sinfónica de Chile (OSCH), Guillermo Rifo (director). 
Albarracín Iribarren, Calatambo (Freddy) (arr. Guillermo Rifo). TM: Cachimbo de Tarapacá (1956), versión para orquesta; F: 9, 10, 15 y 16 de septiembre de 2016; OL: Concierto Nº 17, Música Chilena, Temporada Oficial 2016, Centro de Extensión Artística y Cultural de la Universidad de Chile, Teatro de la Universidad de Chile; Int: Orquesta Sinfónica de Chile, Guillermo Rifo (director).

Alcalde Cordero, Andrés. TM: Atte (2016) para flauta y cuerdas; F: 5 de octubre de 2016; OL: Temporada Oficial, Universidad de Santiago de Chile, Aula Magna de la Universidad de Santiago de Chile (USACH); Int: Diego Vieytes (flauta), Orquesta Clásica de la Universidad de Santiago de Chile, Helmuth Reichel Sila (director).

TM: Sosiega la noche, ópera filopatafísica del ciclo Más allá de la tarde; F: 4 de agosto de 2016; OL: VII Encuentro Internacional de Compositores, Instituto de Música, Facultad de Artes de la Pontificia Universidad Católica de Chile, Corporación Artística de Música Experimental, Matta 365, Viña del Mar; Int: Orquesta y Collegium Vocale, Andrés Alcalde (director).

Alexander Polack, Leni. TM: Divertimento rítmico (1956) para orquesta; F: 3 y 4 de junio de 2016; OL: Orquesta Sinfónica de Chile, 75 años, Centro de Extensión Artística y Cultural de la Universidad de Chile (CEAC), Teatro de la Universidad de Chile; Int: Orquesta Sinfónica de Chile, Helmuth Reichel Silva (director, residente en Alemania).

Allende Sarón, Pedro Humberto (Luis Orlandini). TM: Tonada de carácter popular chileno $\mathrm{N}^{\circ} 5$ (1918), versión para guitarra; F: 26 de abril de 2016; OL: Inauguración del Año Académico 2016 del Instituto de Chile, Auditorio del Instituto de Chile; Int: Luis Orlandini Robert (guitarra).

TM: Tonadas $\mathrm{N}^{\circ} 3, \mathrm{~N}^{\circ} 5, \mathrm{~N}^{\circ} 6$ (de 12 Tonadas de carácter popular chileno) (1918-1922), versión para dos saxofones, marimba y chinchín; F: 19 de mayo de 2016; OL: Academia Chilena de Bellas Artes, Salón de Honor del Instituto de Chile. F: 15 de junio de 2016; OL: Tricahue, percusión y saxofones "Latinoamérica al vuelo", Instituto Cervantes, Varsovia, Polonia. F: 16 de junio de 2016; OL: Tricahue, percusión y saxofones "Latinoamérica al vuelo", Sochaczew, Polonia. F: 18 de junio de 2016; OL: Tricahue, percusión y saxofones "Latinoamérica al vuelo", Instituto Cervantes, de Utrecht, Día del Español. F: 19 de junio de 2016; OL: Tricahue, percusión y saxofones "Latinoamérica al vuelo", Ongekend Bijzonder Festival, Centro "De Heuvel", Rotterdam, Países Bajos. F: 21 de junio de 2016; OL: Tricahue, percusión y saxofones "Latinoamérica al vuelo", VIII Festival LEA Literatura en Atenas, Tecnopolis, Atenas. F: 23 de junio de 2016; OL: Tricahue, percusión y saxofones "Latinoamérica al vuelo", Festival LEA Lefkada, Grecia. Int: Grupo Tricahue: Karem Ruiz (saxofón soprano y barítono), Alejandro Rivas (saxofón soprano y alto), Simone Caiafa (marimba), Olga Carrasco (chinchín y percusiones).

TM: La voz de las calles (1920) para orquesta; F: 6 y 7 de julio de 2016; OL: Temporada Oficial, Concierto $N^{\circ}$ 8; Teatro Municipal de Santiago; Int: Orquesta Filarmónica de Santiago, David Syrus (director). 
Alvarado Gutiérrez, Boris. TM: Ritual wuilliche (2013) para voz, piano, violín, violonchelo y clarinete; F: 11 de agosto de 2016; OL: Festival de Música Contemporánea de Córdoba, Argentina, Salón Auditorio "Arturo Trigueros", Campus Ciudad de las Artes; Int: Eva Luna Navarre (voz), Ximena Medina (piano), Daniel Rivera (violín), Rosel Rojas (violonchelo), Constanza Maletesta (clarinete).

TM: Chamille (2015) para violonchelo; F: 11 de agosto de 2016; OL: Festival de Música Contemporánea de Córdoba, Argentina, Salón Auditorio "Arturo Trigueros", Campus Ciudad de las Artes; Int: Camila Leal (violonchelo).

TM: Gió (2016) para saxofón; F: 11 de agosto de 2016; OL: Festival de Música Contemporánea de Córdoba, Argentina, Salón Auditorio "Arturo Trigueros”, Campus Ciudad de las Artes; Int: Daniela Tolosa (saxofón).

Álvarez Núñez, Pedro. TM: Inherent Nodes (2009) para consort de guitarras; F: 23 de junio de 2016; OL: Concierto de Presentación CD CGCL-Micorriza del Consort Guitarrístico de Chile, Salón de Honor, Pontificia Universidad Católica de Valparaíso; Int: Consort Guitarrístico de Chile.

Aranda, Pablo. TM: *El desvario del apuntador (2016), pieza escénica para piano, marimba, violín, voz y flauta; $F$ : 28 de julio de 2016; OL: VII Encuentro Internacional de Compositores, Instituto de Música, Facultad de Artes de la Pontificia Universidad Católica de Chile, Centro Cultural Gabriela Mistral (GAM), Sala A1; Int: Taller de Música Contemporánea, Pablo Aranda (director).

TM: Espacios que suenan. Performance sonora. Consiste en tres partituras independientes de tres compositores. Cada uno realiza una acción sonora en un piso del estacionamiento, y los asistentes los irán recorriendo en forma ascendente hasta llegar al Goethe Institut; F: 30 de julio de 2016; OL:VII Encuentro Internacional de Compositores, Instituto de Música, Facultad de Artes de la Pontificia Universidad Católica de Chile, Goethe Institut, Int: Pablo Aranda, Antonio Carvallo y Andrés Núñez. Ver además las entradas bajo este título de obras correspondientes a Antonio Carvallo Pinto y Andrés Muñoz Arellano.

TM: Jetzt (1990), pieza para guitarra y flauta; F: 21 de septiembre de 2016; OL: Temporada Oficial de Conciertos 2016, Departamento de Música y Sonología, Facultad de Artes de la Universidad de Chile, Sala Isidora Zegers; Int: DMUS ENSEMBLE (ensemble de profesores): Luis Orlandini (guitarra), Victoria Muñoz (flauta), Andrés Maupoint (director).

Asuar Puigrrós, José Vicente. TM: Lamentos haitianos (1954) para soprano y piano; F: 16 de junio de 2016; OL: Concierto de canto y piano, Academia Chilena de Bellas Artes, Salón de Honor del Instituto de Chile; Int: Camila García (soprano), Jacqueline Urízar (piano).

TM: Formas (1970), música computacional al lenguaje sinfónico; F: 26 y 27 de agosto de 2016; OL: Orquesta Sinfónica de Chile 75 años,Concierto $\mathrm{N}^{\circ} 15$, Centro de Extensión Artística y Cultural de la Universidad de Chile (CEAC), Teatro de la Universidad de Chile; Orquesta Sinfónica de Chile, François López-Ferrer (director, España/Estados Unidos). 
Atria Ramírez, Jaime (arr. Guillermo Rifo Suárez). TM: La consentida (1965), versión para orquesta; $F:$ 9, 10, 15 y 16 de septiembre de 2016; OL: Concierto $\mathrm{N}^{\circ}$ 17, Música Chilena, Temporada Oficial 2016 de la Orquesta Sinfónica de Chile (OSCH), Centro de Extensión Artística y Cultural de la Universidad de Chile (CEAC), Teatro de la Universidad de Chile; Int: Orquesta Sinfónica de Chile, Guillermo Rifo (director).

Becerra Schmidt, Gustavo. TM: Sonata $\mathrm{N}^{\circ} 1$ (1952) para violín y piano; F: 16 de mayo de 2015; OL: Asociación Nacional de Compositores de Chile (ANC) en Otoño, Panorama Contemporáneo Chileno para violín y piano, Sala América, Biblioteca Nacional; Int: Dúo Allendes-Castro: Elías Allendes (violín), Patricia Castro (piano).

TM: Trozo (1961) para trombón tenor; F: 22 de junio de 2016; OL: Proyecto "Desde el Centro 2", Taller No 8, Centro Cultural de Los Andes; Int: Cristián Mezzano (trombón), Francisco Silva (director del proyecto).

TM: Blues, segundo movimiento de la Primera Sonata (1952) para violín y piano; F: 29 de julio de 2016; OL: Concierto de lanzamiento CD Homenaje a compositores chilenos. Piano chileno del siglo XX y XXI, Sala Isidora Zegers, Departamento de Música y Sonología, Facultad de Artes de la Universidad de Chile, Int: Patricia Castro Ahumada (piano), Elías Allendes Osses (violín).

Bianchi Alarcón, Vicente. TM: Variaciones en aire de cueca (1945) para piano; F: 28 de septiembre de 2016; OL: Temporada Oficial, Departamento de Música y Sonología, Facultad de Artes de la Universidad de Chile, Ciclo de piano, Sala Isidora Zegers; Int: Kenya Godoy (piano).

TM: Sajuriando (2000) para piano; F: 28 de septiembre de 2016; OL:Temporada Oficial, Departamento de Música y Sonología, Facultad de Artes de la Universidad de Chile, Ciclo de piano, Sala Isidora Zegers; Int: Kenya Godoy (piano).

TM: Bailando pericona (2010) para piano; F: 28 de septiembre de 2016; OL:Temporada Oficial, Departamento de Música y Sonología, Facultad de Artes de la Universidad de Chile, Ciclo de piano, Sala Isidora Zegers; Int: Kenya Godoy (piano).

Cáceres Romero, Eduardo. TM: Metalmambo (1994), obra electrónica; F: 5 de mayo de 2016; OL: 38th Lueneburg International Seminar Week for Contemporary Music, Glockenhaus, Lüneburg, Alemania, Fortbildungszentrum für neue Musik Lüneburg 2016 (Seminario Internacional de Música Contemporánea, Glockenhaus, Lüneburg, Alemania, Centro de Formación para la Nueva Música).

TM: Feyta (2012) para soprano, kultrún y cascabeles; F: 29 de junio de 2016; OL: SIMUC - Sociedad Internacional para la Música Chilena, Concierto de apertura, Off Theater de Viena, Austria; Int: Clio Montrey (soprano, kultrún y cascabeles).

TM: *Epigramas mapuches (1991) para contralto, clarinete, violín, violonchelo y piano; F: 11 de agosto de 2016; OL: Festival de Música Contemporánea de Córdoba, Argentina, Salón Auditorio “Arturo Trigueros”, Campus Ciudad de las 
Artes; Int: Cecilia Leunda (contralto), Constanza Malatesta (clarinete), Anoush Berberián (violín), Pablo Dolinsky (violonchelo) y Daniel Villegas (piano).

TM: *Huija rendija (2011) para cuarteto de guitarras; F: 11 de agosto de 2016; OL: Festival de Música Contemporánea de Córdoba, Argentina, Salón Auditorio "Arturo Trigueros", Campus Ciudad de las Artes; Int: Cuarteto de Guitarras de Córdoba: Daniela Benavides, Luis Ma. Luján, Fernando Rodríguez, Facundo Figueroa.

TM: *DI cerro Concpcion yo m pac al Alegre...Kiñe. Epu-Kwla..los (2008); F: 21 de septiembre de 2016; OL: Temporada Oficial de Conciertos 2016, Departamento de Música y Sonología, Facultad de Artes de la Universidad de Chile, Sala Isidora Zegers; Int: DMUS ENSEMBLE (ensemble de profesores, Andrés Maupoint (director). F: 22 de septiembre de 2016; OL: Centro Cultural Kirchner de Buenos Aires, Argentina; Int: Ensamble Tropi, Haydée Schvartz (directora).

Cádiz, Rodrigo. TM: Id-Fusiones (2001) para electrónica y medios audiovisuales; F: 25 de mayo de 2016; OL: Temporada Oficial del Departamento de Música y Sonología, Facultad de Artes de la Universidad de Chile, Gabinete de Electroacústica para la Música de Arte (GEMA), Sala Isidora Zegers; Int: Luz María Cury (medios audiovisuales).

Campbell Batista, Ramón. TM: Sonata op. $15 \mathrm{~N}^{\circ} 2$ (1. Allegro molto, 2. Minueto Romanza-Tempo di minueto, 3. Allegro Assai) (1951) para violín y piano; F: 1 de agosto de 2016; OL: Panorama Musical Chileno No 4, Concierto para violín y piano, Sala América de la Biblioteca Nacional; Int: Elías Allendes Osses (violín), Patricia Castro Ahumada (piano).

Candela, José Miguel. TM: El arco de luz (Macarena Aguiló) (2013) para electroacústica sobre soporte (instrumentación, electroacústica); $F: 1$ de julio de 2016; OL: Concierto lanzamiento CD Cuadro piezas acusmáticas por los derechos humanos, Proyecto financiado por la Vicerrectoría de Investigación y Desarrollo de la Universidad de Chile (VID), Sala Sazié, Casa Central de la Universidad de Chile. F: 17 de agosto de 2016; OL: Aula Virtual, Universidad Metropolitana de Ciencias de la Educación (UMCE). F: 26 de agosto de 2016; OL: Concierto, Centro Cultural San Joaquín. F: 7 de septiembre de 2016; OL: Concierto, Centro Cultural El Bosque. F: 9 de septiembre de 2016; OL: Auditorio IMUC (Instituto de Música de la Pontificia Universidad Católica de Chile), Campus Oriente.

TM: Primeros encuentros con la vida y con la muerte (Leopoldo Muñoz) (2014) para electroacústica sobre soporte (instrumentación, electroacústica); $F: 1$ de julio de 2016; OL: Concierto lanzamiento CD Cuadro piezas acusmáticas por los derechos humanos, Proyecto financiado por la Vicerrectoría de Investigación y Desarrollo de la Universidad de Chile (VID), Sala Sazié, Casa Central de la Universidad de Chile. F: 17 de agosto de 2016; OL: Aula Virtual, Universidad Metropolitana de Ciencias de la Educación (UMCE). F: 26 de agosto de 2016; OL: Concierto, Centro Cultural San Joaquín. F: 7 de septiembre de 2016; OL: Concierto, Centro Cultural El Bosque. F: 9 de septiembre de 2016; OL: Auditorio IMUC (Instituto de Música de la Pontificia Universidad Católica de Chile), Campus Oriente. 
TM: Por tus escaleras, no camine el dolor (Humberto Miranda) (2015) para electroacústica sobre soporte (instrumentación, electroacústica); $F: 1$ de julio de 2016; OL: Concierto lanzamiento CD Cuadro piezas acusmáticas por los derechos humanos, Proyecto financiado por la Vicerrectoría de Investigación y Desarrollo de la Universidad de Chile (VID), Sala Sazié, Casa Central de la Universidad de Chile. F: 17 de agosto de 2016; OL: Aula Virtual, Universidad Metropolitana de Ciencias de la Educación (UMCE). F: 26 de agosto de 2016; OL: Concierto, Centro Cultural San Joaquín. F: 7 de septiembre de 2016; OL: Concierto, Centro Cultural El Bosque. F: 9 de septiembre de 2016; OL: Auditorio IMUC (Instituto de Música de la Pontificia Universidad Católica de Chile), Campus Oriente.

TM: Un pequeño acto de resistencia (Ana María Jiménez y Teresa Izquierdo) (2016) para electroacústica sobre soporte (instrumentación, electroacústica); F: 1 de julio de 2016; OL: Concierto lanzamiento CD Cuadro piezas acusmáticas por los derechos humanos, Proyecto financiado por la Vicerrectoría de Investigación y Desarrollo de la Universidad de Chile (VID), Sala Sazié, Casa Central de la Universidad de Chile. F: 17 de agosto de 2016; OL: Aula Virtual, Universidad Metropolitana de Ciencias de la Educación (UMCE) . F: 26 de agosto de 2016; OL: Concierto, Centro Cultural San Joaquín. F: 7 de septiembre de 2016; OL: Concierto, Centro Cultural El Bosque. F: 9 de septiembre de 2016; OL: Auditorio IMUC (Instituto de Música de la Pontificia Universidad Católica de Chile), Campus Oriente.

Candela, José Miguel y Daniel Nieto. TM: $A M E$ (Audiciones de Música Electroacústica) (2016); F: 25 de agosto de 2016; OL: Teatro Centro Cultural Las Condes.

Cantón Aguirre, Edgardo. TM: Introducción-Allegro-Final (1981) para piano; F: 29 de julio de 2016; OL: Concierto de lanzamiento CD Homenaje a compositores chilenos. Piano chileno del siglo XX y XXI, Sala Isidora Zegers, Departamento de Música y Sonología, Facultad de Artes de la Universidad de Chile, Int: Patricia Castro Ahumada.

TM: Atacama imaginario (2009) para orquesta; F: 11 de agosto de 2016; OL: Ciclo Sinfónico Banda Sinfónica Estudiantil, Departamento de Música y Sonología, Facultad de Artes de la Universidad de Chile, Sala Isidora Zegers; Int: Banda Sinfónica Estudiantil del Departamento de Música y Sonología, Eduardo Browne (director).

TM: Balada (1985) para soprano; F: 25 de agosto de 2016; OL: Departamento de Música y Sonología, Facultad de Artes de la Universidad de Chile, Centro Integral Adulto Mayor Diego de Almagro, Ilustre Municipalidad de Providencia, Int: María Graciela Antivero.

TM: De Cir y lo (2015) para flauta, clarinete, violín, violonchelo y piano; F: 21 de septiembre de 2016; OL: Temporada Oficial de Conciertos 2016, Departamento de Música Sonología, Facultad de Artes de la Universidad de Chile, Sala Isidora Zegers; Int: DMUS ENSEMBLE (ensemble de profesores): Wilson Padilla Véliz (flauta), Alejandro Ortiz (clarinete), Elías Allendes (violín), Julio Barrios (violochelo), Andrés Maupoint (piano, director). 
Cárdenas Vargas, Félix. TM: Rito (2002) para flauta; F: 1 de junio de 2016; OL: Temporada Oficial del Departamento de Música y Sonología, Facultad de Artes de la Universidad de Chile, Fundación Cultural de Providencia; Int: Wilson Padilla Véliz (flauta). F: 11 de agosto de 2016; OL: Festival de Música Contemporánea de Córdoba, Argentina, Salón Auditorio “Arturo Trigueros”, Campus Ciudad de las Artes; Int: Laura Pinto (flauta).

Carnicer Batlle, Ramón. TM: Himno Nacional de Chile (1828-1847) para voz y piano, texto de Eusebio Lillo; F: 29 de septiembre de 2016; OL: Ceremonia de celebración de los 52 años del Instituto de Chile, Salón de Honor del Instituto de Chile; Int: Francisco Huerta (tenor), Patricia Castro Ahumada (piano).

Carrasco Pantoja, Fernando. TM: VIP (2000) para piano; F: 29 de julio de 2016; OL: Concierto de lanzamiento CD Homenaje a compositores chilenos. Piano chileno del siglo XX y XXI, Sala Isidora Zegers, Departamento de Música y Sonología, Facultad de Artes de la Universidad de Chile; F: 8 de agosto de 2016; OL: Temporada Oficial de Conciertos, Departamento de Música y Sonología, Facultad de Artes de la Universidad de Chile, Ciclo Música Chilena y Latinoamericana, Biblioteca Nacional; Int: Patricia Castro Ahumada (piano).

Carrasco Pirard, Eduardo/ Hernán Gómez. TM: Patria de multitudes (1976) para conjunto vocal e instrumental; F: 21 de abril de 2016; OL: Celebración de los 50 años del conjunto Quilapayún, Teatro Municipal de Santiago; Int: Conjunto Quilapayún junto a la Orquesta Sinfónica Estudiantil Metropolitana, Fernando Saavedra (director).

Carvallo Pinto, Antonio. TM: Imagen en el espejo (2012), versión para voz recitante, medios electrónicos y visuales; $F$ : 25 de mayo de 2016; OL: Temporada Oficial del Departamento de Música y Sonología, Facultad de Artes de la Universidad de Chile, Gabinete de Electroacústica para la Música de Arte (GEMA), Sala Isidora Zegers; Int: Paulina Lorca (voz recitante), Isabel Pérez del Pulgar (medios audiovisuales).

F: Berceuse (2014) para vibráfono, arpa y piano; F: 6 de julio de 2016; OL: Temporada Oficial del Departamento de Música y Sonología, Facultad de Artes de la Universidad de Chile, Ciclo Siglo XIX y XX, Música instrumental contemporánea, Sala Isidora Zegers; Int: Gad Xoyon (vibráfono), Pablo Cristi (arpa), Jorge Pepi-Alos (piano).

F: Tres piezas (2000) para piano y violín; F: 29 de julio de 2016; OL: Concierto de lanzamiento CD Homenaje a compositores chilenos. Piano chileno del siglo XX y XXI, Sala Isidora Zegers, Departamento de Música y Sonología, Facultad de Artes de la Universidad de Chile; Int: Patricia Castro Ahumada (piano), Elías Allendes Osses (violín).

TM: Espacios que suenan. Performance sonora. Consiste en tres partituras independientes de tres compositores. Cada uno realiza una acción sonora en un piso del estacionamiento, y los asistentes los irán recorriendo en forma ascendente hasta llegar al Goethe Institut; F: 30 de julio de 2016; OL:VII Encuentro Internacional de Compositores, Instituto de Música, Facultad de Artes de la Pontificia Universidad 
Católica de Chile, Goethe Institut; Int: Pablo Aranda, Antonio Carvallo y Andrés Núñez. Ver además las entradas bajo este título de obras correspondientes a Pablo Aranda y Andrés Muñoz Arellano.

TM: Elipses (2011) para bailarina y electrónica en tiempo real; F: $10 \mathrm{de}$ agosto de 2016; OL: Temporada Oficial, Departamento de Música y Sonología, Facultad de Artes de la Universidad de Chile, Ciclo Siglo XX y XXI, Gema (Gabinete de Electroacústica para la Música de Arte), Sala Isidora Zegers; Int: Lilian Pizarro (bailarina).

Castellón, Guillermo. TM: Meditación 2 (Lento misterioso) (1981) para fagot y piano, obra dedicada a Jorge Espinoza Flores; F: 8 de agosto de 2016; OL: Temporada Oficial de Conciertos 2016, Departamento de Música y Sonología, Facultad de Artes de la Universidad de Chile, Ciclo Música Chilena y Latinoamericana, Biblioteca Nacional; Int: Jorge Espinoza (fagot), Patricia Castro Ahumada (piano).

Chávez Chávez, Luis. TM: Loncomilla Choique Purrún (2014), segundo lugar en el X Concurso de Composición Musical Luis Advis 2014; F: 15 de septiembre de 2016; OL: Concierto para guitarra, La Maison de l'Amérique Latine (Casa de América Latina), París, Francia; Int: César Farías Huenuqueo (guitarra).

Contreras, Javier. TM: Tonada funcional $\mathrm{N}^{\circ} 7$ (2013) para guitarra; F: 28 de septiembre de 2016; OL: Departamento de Música y Sonología, Facultad de Artes de la Universidad de Chile, Concierto Cátedra profesor Romilio Orellana, Escuela Salvador Sanfuentes; Int: Javier Contreras.

Contreras Vázquez, Manuel. TM: *Moebius (2015-2016), ópera de cámara para 3 voces femeninas y ensamble de 12 instrumentos, obra sobre el tema de la inmigración, libreto de Manuel Contreras; F: 27 de agosto de 2016; OL: Parque Cultural de Valparaíso. F: 2 de septiembre de 2016; OL: Centro Cultural Gabriela Mistral (GAM); Int: Nancy Gómez, Cecilia Barrientos, Camila García (sopranos), Sidhartha Corvalán (Régie), Gonzalo Venegas (dirección musical), Ensamble Instrumental FOJI, Laura Bisotti (escenografía), Elías Sepúlveda (iluminación, Instrumental Producciones, realización audiovisual), José Oplustil (sonido), Orietta Cabezas (diseño gráfico). Proyecto financiado por el Fondo de la Música Nacional, Agencia Española de Cooperación, Fundación de Orquestas Juveniles e Infantiles de Chile (FOJI), Fundación Kone de Finlandia, Instituto Italiano de Cultura, Estudio Modular de Música Actual, Almamia Atelier, Agrupación Amigos de la Ópera de Antofagasta e Instituto de Música de la Pontificia Universidad Católica de Chile.

Correa Astudillo, Esteban. TM: *Animal de umbrales (2016) para oboe y orquesta; F: 5 de abril de 2016; OL: Centro de Eventos Joaquín Blaya, Curacaví. F: 6 de abril de 2016; OL: Parroquia La Transfiguración del Señor, Las Condes. F: 7 de abril de 2016; OL: Hall Central Edificio Consistorial Municipal Estación Central. F: 8 de abril de 2016; OL: Teatro Municipal de Nuñoa; Int: José Luis Urquieta Plaza (oboe), Orquesta de Cámara de Chile, Vicente Larrañaga (director).

Cortés Ahumada, Nicolás. TM: Apu Wamani (2015) para tuba y electrónica; F: 25 de mayo de 2016; OL: Temporada Oficial del Departamento de Música y Sonología, 
Facultad de Artes de la Universidad de Chile, Gabinete de Electroacústica para la Música de Arte (GEMA), Sala Isidora Zegers; Int: Carlos Herrera (tuba).

De Negri Murillo, Fabrizzio. TM: *Fantasía concertante (2015) para oboe y cinta; F: 2 de mayo de 2016; OL: Presentación del disco Nuevos aires chilenos para oboe, Salón Mecesup, Departamento de Música de la Universidad de La Serena. F: 9 de mayo de 2016; OL: Presentación del disco Nuevos aires chilenos para oboe, Teatro Caixa Preta, Universidad Federal de Santa María, Brasil; Int: José Luis Urquieta Plaza (oboe).

Délano Thayer, Pablo. TM: Antigua balada (2015) para violín y piano, obra dedicada al Dúo Allendes-Castro; F: 16 de mayo de 2015; OL: Asociación Nacional de Compositores de Chile (ANC) en Otoño, Panorama contemporáneo chileno para violín y piano, Sala América, Biblioteca Nacional; Int: Dúo Allendes-Castro: Elías Allendes (violín), Patricia Castro (piano).

TM: Danza 1970 (1970) para piano; F: 9 de septiembre de 2016; OL: Casa de la Cultura Anselmo Cádiz, Comuna El Bosque; Int: Bastián Jorquera (piano).

Errandonea, Cristián. TM: Olvídame (2006) para conjunto; F: 19 de mayo de 2016; OL: Academia Chilena de Bellas Artes, Salón de Honor del Instituto de Chile. F: 15 de junio de 2016; OL: Tricahue, percusión y saxofones "Latinoamérica al vuelo", Instituto Cervantes, Varsovia, Polonia. F: 16 de junio de 2016; OL: Tricahue, percusión y saxofones "Latinoamérica al vuelo", Sochaczew, Polonia. F: 18 de junio de 2016; OL: Tricahue, percusión y saxofones "Latinoamérica al vuelo", Instituto Cervantes, de Utrecht, Día del Español, Tricahue, percusión y saxofones "Latinoamérica al vuelo". F: 19 de junio de 2016; OL: Tricahue, percusión y saxofones "Latinoamérica al vuelo", Ongekend Bijzonder Festival, Centro "De Heuvel", Rotterdam, Países Bajos. F: 21 de junio de 2016; OL: Tricahue, percusión y saxofones "Latinoamérica al vuelo", VIII Festival LEA Literatura en Atenas, Tecnopolis, Atenas. F: 23 de junio de 2016; OL: Tricahue, percusión y saxofones "Latinoamérica al vuelo", Festival LEA Lefkada, Grecia; Int: Grupo Tricahue: Karem Ruiz (saxofón soprano y barítono), Alejandro Rivas (saxofón soprano y alto), Simone Caiafa (marimba), Cristián Errandonea (contrabajo), Miguel Zárate (batería y percusiones), Olga Carrasco (chinchín y percusiones).

TM: CRSK (2014) para conjunto; F: 19 de mayo de 2016; OL: Academia Chilena de Bellas Artes, Salón de Honor del Instituto de Chile. F: 15 de junio de 2016; OL: Tricahue, percusión y saxofones "Latinoamérica al vuelo", Instituto Cervantes, Varsovia, Polonia. F: 16 de junio de 2016; OL: Tricahue, percusión y saxofones "Latinoamérica al vuelo", Sochaczew, Polonia. F: 18 de junio de 2016; $O L$ : Tricahue, percusión y saxofones "Latinoamérica al vuelo", Instituto Cervantes, de Utrecht, Día del Español, Tricahue, percusión y saxofones "Latinoamérica al vuelo". F: 19 de junio de 2016; OL: Tricahue, percusión y saxofones "Latinoamérica al vuelo", Ongekend Bijzonder Festival, Centro "De Heuvel”, Rotterdam, Países Bajos. F: 21 de junio de 2016; OL: Tricahue, percusión y saxofones "Latinoamérica al vuelo", VIII Festival LEA Literatura en Atenas, Tecnopolis, Atenas. F: 23 de junio de 2016; OL: Tricahue, percusión y saxofones "Latinoamérica al vuelo", Festival LEA Lefkada, Grecia. Int: Grupo Tricahue: Karem Ruiz (saxofón soprano 
y barítono), Alejandro Rivas (saxofón soprano y alto), Simone Caiafa (marimba), Cristián Errandonea (contrabajo), Miguel Zárate (batería y percusiones), Olga Carrasco (chinchín y percusiones).

Errázuriz Rodríguez, Sebastián. TM: *Música coreográfica sobre las Variaciones Goldberg de Johann Sebastian Bach (2016) para piano, violonchelo, violín y viola; F: 22 de julio de 2016; OL: Ciclo No 2- Danza y Música, Estreno de la Coreografía Dos veces Bach, Centro de Extensión Artística y Cultural de la Universidad de Chile, Teatro de la Universidad de Chile; Int: Integrantes de la Orquesta Sinfónica de Chile: Luis Alberto Latorre (piano), Juan Goic (violonchelo), Miguel Ángel Muñoz (violín), Claudio Gutiérrez (viola). Ballet Nacional Chileno y bailarines invitados de Ballet de Santiago, Mathieu Guilhaumon (coreógrafo, Francia).

Farías Caballero, Javier. TM: *La oración para todos (2016) para ensamble de guitarras y coro, texto basado en el poema homónimo de Andrés Bello; F: 11 de agosto de 2016; OL: Dirección de Extensión Cultural de la Universidad Andrés Bello (UNAB), Salón Colonial del Campus Casona Las Condes; Int: Ensamble de Guitarras de Chile, Javier Farías (director), Coro de la Universidad Andrés Bello, Jessica Quezada (directora).

TM: El adiós en forma de cueca (2008); F: 15 de septiembre de 2016; OL: Concierto para guitarra, La Maison de l'Amérique Latine (Casa de América Latina), París, Francia; Int: César Farías Huenuqueo (guitarra).

Farías Vásquez, Miguel. TM: *Continuo (2008) para oboe; F: 6 de mayo de 2016; OL: Presentación del disco Nuevos aires chilenos para oboe, Auditorio, Instituto de Chile. F: 9 de mayo de 2016; OL: Presentación del disco Nuevos aires chilenos para oboe, Teatro Caixa Preta, Universidad Federal de Santa María, Brasil; Int: José Luis Urquieta Plaza (oboe).

TM: Estudio $\mathrm{N}^{\circ} 8$ (2013) para piano; F: 27 de agosto de 2016; OL: Inauguración oficial de la sala de conciertos Arthémuse. Art-Théâtre-Musique, París, Francia; Int: María Paz Santibáñez (piano).

*Voz de piedra (versión revisada 2016) para orquesta de cámara, escrita especialmente para la Orquesta de Cámara y Rodolfo Fischer; F: 13 de septiembre de 2016; OL: Gimnasio Liceo Zapallar, Las Condes. F: 14 de septiembre de 2016; OL: Parroquia La Transfiguración del Señor, Las Condes. F: 15 de septiembre de 2016; OL: Teatro Oriente, Providencia. F: 16 de septiembre de 2016; OL: Teatro Municipal de Nuñoa; Int: Orquesta de Cámara de Chile, Rodolfo Fischer (director invitado), Alejandra Urrutia (directora titular).

Faró, Chito [Enrique Motto Arenas] (arr. Guillermo Rifo). TM: Si vas para Chile (1942), versión para orquesta; F: 9, 10, 15 y 16 de septiembre de 2016; OL: Concierto N $^{\circ} 17$, Música Chilena, Temporada Oficial 2016 de la Orquesta Sinfónica de Chile (OSCH), Centro de Extensión Artística y Cultural de la Universidad de Chile, Teatro de la Universidad de Chile; Int: Orquesta Sinfónica de Chile, Guillermo Rifo (director). 
Focke, Fré. TM: Le tombeau de Van Gogh (20 piezas para piano) (1951); F: 6 de julio de 2016; OL: Temporada Oficial Departamento de Música y Sonología, Facultad de Artes de la Universidad de Chile, Ciclo Siglo XIX y XX, Música instrumental contemporánea, Sala Isidora Zegers. F: 21 de septiembre de 2016; OL: Temporada Oficial de Conciertos 2016, Departamento de Música y Sonología, Facultad de Artes de la Universidad de Chile, Sala Isidora Zegers; Int: Andrés Maupoint (piano).

Frigerio, Aída. TM: Los aliados (siglo XIX) para piano; F: 28 de septiembre de 2016; OL: Temporada Oficial, Departamento de Música y Sonología, Facultad de Artes de la Universidad de Chile, Ciclo de piano, Sala Isidora Zegers; Int: Kenya Godoy (piano).

Gallardo, Felipe. TM: Puririy (palabra en quechua que significa "ponerse en camino, irse”) (2015) para conjunto instrumental; F: 26 de abril de 2016; OL: Concierto gratuito, Librería Le Monde Diplomatique, Santiago; Int: Grupo Sexto Piso, Claudio Acevedo (director).

García Arancibia, Fernando. TM: Más comentarios sobre dichos populares (2013) para violín y piano; F: 16 de mayo de 2016; OL: Asociación Nacional de Compositores de Chile (ANC) en otoño, Panorama contemporáneo chileno para violín y piano, Sala América, Biblioteca Nacional; Int: Dúo Allendes-Castro: Elías Allendes (violín), Patricia Castro (piano).

TM: Desde la otra orilla (1. Rápido, con furia, 2. Lento, meditativo, 3. LentoRápido-Lento-Rápido) (2014) para orquesta de flautas; F: 17 de mayo de 2016; OL: Temporada Oficial del Departamento de Música y Sonología, Facultad de Artes de la Universidad de Chile, Centro Cultural Gabriela Mistral. F: 25 de mayo de 2016; OL: Semana de la Educación, Concierto educativo Orquesta de Flautas Illawara, Departamento de Música y Sonología, Facultad de Artes de la Universidad de Chile, Sala Isidora Zegers. F: 23 de agosto de 2016; OL: Concierto didáctico, Departamento de Música y Sonología, Facultad de Artes de la Universidad de Chile, Centro Cultural Gabriela Mistral (GAM); Int: Orquesta de Flautas Illawara, Wilson Padilla Véliz (director).

TM: *Palabras de amigos (1. El gato gris, 2. Miro esa nube, 3. Séptima señal, 4. Un mueble que sufre) (2015) para soprano y piano; F: 16 de junio de 2016; OL: Concierto de canto y piano, Academia Chilena de Bellas Artes, Salón de Honor del Instituto de Chile; Int: Camila García (soprano), Jacqueline Urízar (piano).

TM: Cuatro trozos (2016) para trombón, obra dedicada a Cristián Mezzano; F: 22 de junio de 2016; OL: Proyecto "Desde el Centro 2", Taller N ${ }^{\circ}$ 8, Centro Cultural de Los Andes; Int: Cristián Mezzano (trombón), Francisco Silva (director del proyecto).

TM: Ante la duda, abstente, tres nocturnos (1. Lento misterioso: ¿Por qué?, 2. Lentamente y expresivo: ¿Para qué?, 3. Calmo, con dolor: ¿̨ómo?) (2012) para cuarteto de cuerdas, F: 14 de julio de 2016; OL: Proyecto Rescate Patrimonial Chile-Perú, Teatro de la Cultura Peruano Norteamericano, Arequipa, Perú; Int: Cuarteto Surkos. 
TM: Cuatro piezas breves (1. Lento, 2. Rápido, 3. Lento, 4. Rápido) (1985) para violín y piano; F: 1 de agosto de 2016; OL: Panorama Musical Chileno $\mathrm{N}^{\mathrm{o}} 4$, Concierto para violín y piano, Sala América de la Biblioteca Nacional; Int: Elías Allendes Osses (violín), Patricia Castro Ahumada (piano).

TM: Hagamos un trato para recordar (1. Rápido, 2. Lento, 3 Ágil y tenso) (2013) para violín; F: 1 de agosto de 2016; OL: Panorama Musical Chileno $\mathrm{N}^{\mathrm{o}} 4$, Concierto para violín y piano, Sala América de la Biblioteca Nacional; Int: Elías Allendes Osses (violín).

Garrido Lecca, Celso. TM: Simpay (1. Agitado, 2. Calmo, sin rigor, 3. Rústico con vigor) (1988) para guitarra; F: 24 de julio de 2016; OL: Ciclo de Guitarra, Referentes y estrenos, Departamento de Música y Sonología, Facultad de Artes de la Universidad de Chile, Museo Nacional de Bellas Artes, Int: Luis Orlandini (guitarra).

González, Andrés. TM: Aion (2008-2013) para consort de guitarras y ensamble; F: 23 de junio de 2016; OL: Concierto de presentación CD CGCL-Micorriza del Consort Guitarrístico de Chile, Salón de Honor, Pontificia Universidad Católica de Valparaíso; Int: Consort Guitarrístico de Chile y Ensamble f(r)actura.

González, Marcia. TM: La balacera (2013) para conjunto vocal e instrumental; F: 26 de abril de 2016; OL: Concierto gratuito, Librería Le Monde Diplomatique, Santiago; Int: Grupo Sexto Piso, Claudio Acevedo (director).

TM: Como anoche (2015) para conjunto vocal e instrumental; F: 26 de abril de 2016; OL: Concierto gratuito, Librería Le Monde Diplomatique, Santiago; Int: Grupo Sexto Piso, Claudio Acevedo (director).

TM: Moldéala (2015) para conjunto vocal e instrumental; F: 26 de abril de 2016; OL: Concierto gratuito, Librería Le Monde Diplomatique, Santiago; Int: Grupo Sexto Piso, Claudio Acevedo (director).

Guarello Finlay, Alejandro. TM: Estreliano (2010) para violín y piano; F: 11 de agosto de 2016; OL: Festival de Música Contemporánea de Córdoba, Argentina, Salón Auditorio "Arturo Trigueros", Campus Ciudad de las Artes; Int: Carina Cabana (violín), Ma. Luz Cordeiro (piano).

Guillier, Alejandro. TM: Boreal (2014) para dos saxofones, marimba, contrabajo y batería, F: 19 de mayo de 2016; OL: Academia Chilena de Bellas Artes, Salón de Honor del Instituto de Chile. F: 15 de junio de 2016; OL: Tricahue, percusión y saxofones "Latinoamérica al vuelo", Instituto Cervantes, Varsovia, Polonia. F: 16 de junio de 2016; OL: Tricahue, percusión y saxofones "Latinoamérica al vuelo", Sochaczew, Polonia. F: 18 de junio de 2016; OL: Tricahue, percusión y saxofones "Latinoamérica al vuelo", Instituto Cervantes, de Utrecht, Día del Español, Tricahue, percusión y saxofones "Latinoamérica al vuelo". F: 19 de junio de 2016; $O L$ : Tricahue, percusión y saxofones "Latinoamérica al vuelo", Ongekend Bijzonder Festival, Centro "De Heuvel”, Rotterdam, Países Bajos. F: 21 de junio de 2016; $O L$ : Tricahue, percusión y saxofones "Latinoamérica al vuelo", VIII Festival LEA Literatura en Atenas, Tecnopolis, Atenas. F: 23 de junio de 2016; OL: Tricahue, 
percusión y saxofones "Latinoamérica al vuelo", Festival LEA Lefkada, Grecia. Int: Grupo Tricahue: Karem Ruiz (saxofón soprano y barítono), Alejandro Rivas (saxofón soprano y alto), Simone Caiafa (marimba), Cristián Errandonea (contrabajo), Miguel Zárate (batería y percusiones).

Heinlein Funcke, Federico. TM. Calle sombreada de sauces (Quietud) (1944) para voz y piano; F: 1 de septiembre de 2016; OL: Concierto de Música Vocal de Chile y Latinoamérica, Academia Chilena de Bellas Artes, Instituto de Chile; Int: Ismael Latrach (bajo), Patricia Castro Ahumada (piano).

TM: Vida Mía (1946) para voz y piano; F: 1 de septiembre de 2016; OL: Concierto de Música Vocal de Chile y Latinoamérica, Academia Chilena de Bellas Artes, Instituto de Chile; Int: Ismael Latrach (bajo), Patricia Castro Ahumada (piano).

Herrera Muñoz, Rodrigo. TM: *La colina danesa (2015) para trío de vientos; $F: 15$ de junio de 2016; OL: Conciertos de cámara de la Orquesta Sinfónica de Chile, Centro Cultural Gabriela Mistral; Int: Ensamble de Vientos Víctor Tevah: Carmen Almarza (flauta), Rodrigo Herrera Carmona (oboe y corno inglés), Kathya Galleguillos (clarinete y clarinete bajo), Ricardo Aguilera (corno) y Efraín Vidal (fagot y contrafagot).

Isamitt Alarcón, Carlos. TM: Friso araucano (Canción para dormir al niñito, Canto de soltera) (1931); F: 1 de septiembre de 2016; OL: Concierto de Música Vocal de Chile y Latinoamérica, Academia Chilena de Bellas Artes, Instituto de Chile; Int: Gabriela Dossow ( soprano), Patricia Castro Ahumada (piano).

Jara Martínez, Víctor. TM: Manifiesto (1973), versión para cuarteto de guitarras; F: 26 de agosto de 2016; OL: Concierto didáctico "Cuartetos para un Nuevo Tiempo", Liceo N ${ }^{\circ} 1$ de Niñas; Int: Cuarteto de Guitarras Movimientos.

(arr. Guillermo Rifo). TM: El cigarrito (1964), versión para orquesta; F: 9, 10, 15 y 16 de septiembre de 2016; OL: Concierto $\mathrm{N}^{\circ} 17$, Música Chilena, Temporada Oficial 2016 de la Orquesta Sinfónica de Chile (OSCH), Centro de Extensión Artística y Cultural de la Universidad de Chile, Teatro de la Universidad de Chile; Int: Orquesta Sinfónica de Chile, Guillermo Rifo (director).

(arr. Guillermo Rifo). TM: Luchín (1972), versión para orquesta; F: 9, 10, 15 y 16 de septiembre de 2016; OL: Concierto No 17, Música Chilena, Temporada Oficial 2016 de la Orquesta Sinfónica de Chile (OSCH), Centro de Extensión Artística y Cultural de la Universidad de Chile, Teatro de la Universidad de Chile; Int: Orquesta Sinfónica de Chile, Guillermo Rifo (director).

Jorquera Figueroa, Bastián. TM: Pieza $\mathrm{N}^{\circ} 1$ (2016) para piano; F: 9 de septiembre de 2016; OL: Casa de la Cultura Anselmo Cádiz, Comuna El Bosque; Int: Bastián Jorquera (piano).

Julio, Fernando. TM: El ciclo de una obsesión (2014) para consort de guitarras; F: 23 de junio de 2016; OL: Concierto de Presentación CD CGCL-Micorriza del Consort Guitarrístico de Chile, Salón de Honor, Pontificia Universidad Católica de Valparaíso; Int: Consort Guitarrístico de Chile. 
Kliwadenko. Nicolás. TM: Ilk değil ve son değil (2015) para medios electrónicos y piano; F: 25 de mayo de 2016; OL Temporada Oficial del Departamento de Música y Sonología, Facultad de Artes de la Universidad de Chile, Gabinete de Electroacústica para la Música de Arte, Sala Isidora Zegers; Int: Taller Ciclo: Diego Behncke (samples), Pablo Garretón (samples, procesamiento), Nicolás Kliwadenko (samples, feedback), Francisco Martínez (guitarra eléctrica, procesamiento), Julio Torres (piano).

Laiseca, Rafaela. TM: La Constitución (siglo XIX) para piano; F: 28 de septiembre de 2016; OL: Temporada Oficial, Departamento de Música y Sonología, Facultad de Artes de la Universidad de Chile, Ciclo de piano, Sala Isidora Zegers; Int: Kenya Godoy (piano).

Leal, Camila. TM: Mali (2016) para violonchelo; F: 11 de agosto de 2016; OL: Festival de Música Contemporánea de Córdoba, Argentina, Salón Auditorio "Arturo Trigueros", Campus Ciudad de las Artes; Int: Camila Leal (violonchelo).

Leng Haygus, Alfonso. TM: Andante (1922) para cuerdas; F: 22 de abril de 2016; OL: Concierto homenaje que brindó la Fundación de Orquestas Juveniles e Infantiles (FOJI) al ex Presidente Patricio Aylwin, Catedral Metropolitana de Santiago; Int: Orquesta Sinfónica Nacional Juvenil (OSNJ), Rodolfo Fischer (director). F: 26 de mayo de 2016; OL: 3er Concierto de Extensión, Temporada Oficial, Choapa, Limarí, Elqui. F: 27 de mayo de 2016; OL: 3er Concierto de la Temporada Oficial, Teatro Municipal de La Serena; Int: Orquesta Sinfónica de La Serena, Nicolas Rauss (director-Suiza). F: 20 de agosto de 2016; OL: Teatro Municipal de Nuñoa; Int: Orquesta Sinfónica Estudiantil Metropolitana, Paolo Bortolameolli (director).

TM: Canto de invierno (1933) para orquesta; F: 18 y 19 de mayo de 2016; OL: Temporada Oficial, Concierto $\mathrm{N}^{\circ}$ 7, El Romanticismo Orquestado, Teatro Municipal de Santiago; Int: Orquesta Filarmónica de Santiago, Konstantin Chudovsky (director).

TM: Lass meine Tränen fliessen (1918) para voz y piano; $F$ : 1 de septiembre de 2016; OL: Concierto de Música Vocal de Chile y Latinoamérica, Academia Chilena de Bellas Artes, Instituto de Chile; Int: Carolina Muñoz (soprano), Patricia Castro Ahumada (piano).

TM: Vigilien (1955) para voz y piano; F: 1 de septiembre de 2016; OL: Concierto de Música Vocal de Chile y Latinoamérica, Academia Chilena de Bellas Artes, Instituto de Chile; Int: Carolina Muñoz (soprano), Patricia Castro Ahumada (piano).

Letelier Llona, Alfonso. TM: Sonatina (1953) para violín y piano; F: 16 de mayo de 2015; OL: Asociación Nacional de Compositores de Chile (ANC) en Otoño, Panorama Contemporáneo Chileno para violín y piano, Sala América, Biblioteca Nacional; Int: Dúo Allendes-Castro: Elías Allendes (violín), Patricia Castro (piano).

TM: Suite grotesca (Entrada-vals-Interludio digestivo - La mona triste) (1936) para violín y piano; F: 29 de julio de 2016; OL: Concierto de lanzamiento CD Homenaje a compositores chilenos. Piano chileno del siglo XX y XXI, Sala Isidora Zegers, 
Departamento de Música y Sonología, Facultad de Artes de la Universidad de Chile. F: 1 de agosto de 2016; OL: Panorama Musical Chileno $\mathrm{N}^{\circ}$ 4, Concierto para violín y piano, Sala América de la Biblioteca Nacional; Int: Elías Allendes Osses (violín), Patricia Castro Ahumada (piano).

TM: Madrigal (1933-1934) para voz y piano; $F$ : 1 de septiembre de 2016; OL: Concierto de Música Vocal de Chile y Latinoamérica, Academia Chilena de Bellas Artes, Instituto de Chile; Int: Ismael Latrach (bajo), Patricia Castro Ahumada (piano).

Letelier Valdés, Miguel. TM: A Julio Perceval (Tempo de chamamé. Rubato) (1995) para piano; F: 29 de julio de 2016; OL: Concierto de lanzamiento CD Homenaje a compositores chilenos. Piano chileno del siglo XX y XXI, Sala Isidora Zegers, Departamento de Música y Sonología, Facultad de Artes de la Universidad de Chile. F: 8 de agosto de 2016; OL: Temporada Oficial de Conciertos 2016, Departamento de Música y Sonología, Facultad de Artes, Ciclo Música Chilena y Latinoamericana, Biblioteca Nacional; Int: Patricia Castro Ahumada.

TM: A Violeta Parra (Tempo de tonada. Tranquilo y algo lángido) (1995) para piano; F: 29 de julio de 2016; OL: Concierto de lanzamiento CD Homenaje a compositores chilenos. Piano chileno del siglo XX y XXI, Sala Isidora Zegers, Departamento de Música y Sonología, Facultad de Artes de la Universidad de Chile. (Tempo de tonada); F: 8 de agosto de 2016; OL: Temporada Oficial de Conciertos 2016, Departamento de Música y Sonología, Facultad de Artes de la Universidad de Chile, Ciclo Música Chilena y Latinoamericana, Biblioteca Nacional; Int: Patricia Castro Ahumada.

TM: Alguien pasó (Tempo giusto) (1995) para piano; F: 29 de julio de 2016; OL: Concierto de lanzamiento CD Homenaje a compositores chilenos. Piano chileno del siglo XX y XXI, Sala Isidora Zegers, Departamento de Música y Sonología, Facultad de Artes de la Universidad de Chile, Int: Patricia Castro Ahumada. F: 8 de agosto de 2016; OL: Temporada Oficial de Conciertos, Departamento de Música y Sonología, Facultad de Artes de la Universidad de Chile, Ciclo Música Chilena y Latinoamericana, Biblioteca Nacional; Int: Patricia Castro Ahumada (piano).

TM: Tiempos idos (Lento molto espresivo) (1995) para piano; F: 8 de agosto de 2016; OL: Temporada Oficial de Conciertos 2016, Departamento de Música y Sonología, Facultad de Artes de la Universidad de Chile, Ciclo Música Chilena y Latinoamericana, Biblioteca Nacional; Int: Patricia Castro Ahumada (piano).

Los Jaivas (arr. Guillermo Rifo Suárez). TM: Todos juntos (ca. 1971), versión para orquesta; F: 9, 10, 15 y 16 de septiembre de 2016; OL: Concierto $\mathrm{N}^{\circ} 17$, Música Chilena, Temporada Oficial 2016, Centro de Extensión Artística y Cultural de la Universidad de Chile, Teatro Universidad de Chile, Int: Orquesta Sinfónica de Chile, Guillermo Rifo (director).

Manns, Patricio (arr. Guillermo Rifo Suárez). TM: Balada de los amantes del camino de Taverney (1983), versión para orquesta; F: 9, 10, 15 y 16 de septiembre de 2016; OL: Concierto $\mathrm{N}^{\circ}$ 17, Música Chilena, Temporada Oficial de la Orquesta Sinfónica de Chile (OSCH), Centro de Extensión Artística y Cultural de la Universidad de 
Chile, Teatro Universidad de Chile; Int: Orquesta Sinfónica de Chile, Guillermo Rifo (director).

(arr. Guillermo Rifo Suárez). TM: Arriba en la cordillera (1965), versión para orquesta; F: 9,10, 15 y 16 de septiembre de 2016; OL: Concierto $\mathrm{N}^{\mathrm{o}} 17$, Música Chilena, Temporada Oficial 2016 de la Orquesta Sinfónica de Chile (OSCH), Centro de Extensión Artística y Cultural de la Universidad de Chile, Teatro de la Universidad de Chile; Int: Orquesta Sinfónica de Chile, Guillermo Rifo (director).

(arr. Guillermo Rifo Suárez). TM: Llegó volando (1983), versión para orquesta; F: 9, 10, 15 y 16 de septiembre de 2016; OL: Concierto $\mathrm{N}^{\circ} 17$, Música Chilena, Temporada Oficial de la Orquesta Sinfónica de Chile (OSCH), Centro de Extensión Artística y Cultural de la Universidad de Chile, Teatro de la Universidad de Chile; Int: Orquesta Sinfónica de Chile, Guillermo Rifo (director).

Márquez, Roberto/Nelly Lemus (arr. Guillermo Rifo Suárez). TM: Morena esperanza (ca. 1997), versión para orquesta, texto de Nicolás Guillén; F: 9, 10, 15 y 16 de septiembre de 2016; OL: Concierto N 17, Música Chilena, Temporada Oficial 2016 de la Orquesta Sinfónica de Chile (OSCH), Centro de Extensión Artística y Cultural de la Universidad de Chile, Teatro de la Universidad de Chile, Teatro de la Universidad de Chile; Int: Orquesta Sinfónica de Chile, Guillermo Rifo (director).

Martínez Serrano, Jorge. TM: La rosa con el clavel (1962), versión para quinteto de bronces; F: 26 de mayo de 2016; OL: Semana de la Educación, Concierto Educativo Bill Brass, Departamento de Música y Sonología, Facultad de Artes de la Universidad de Chile, Sala Isidora Zegers; Int: Quinteto de Bronces Bill Brass: Wilson García (trompeta), Pablo Carreño (trompeta), Sebastián Lizana (corno francés), David Naranjo (trombón) y Nicolás Cortés (tuba), Claudio Merino (comentarios).

Martínez Ulloa, Jorge. TM: Cuando faltan las palabras (2015) para violín y piano, obra dedicada al Dúo Allendes-Castro; F: 16 de mayo de 2016; OL: Asociación Nacional de Compositores de Chile (ANC) en otoño, Panorama contemporáneo chileno para violín y piano, Sala América, Biblioteca Nacional; Int: Dúo AllendesCastro: Elías Allendes (violín), Patricia Castro (piano).

TM: Tres piezas latinoamericanas (1. Andino, 2. Coral, 3. Son) (2013) para guitarra; F: 24 de mayo de 2016; OL: Semana de la Educación Artística. Un artista en mi escuela, Vicerrectoría de Extensión de la Universidad de Chile, Liceo Ciudad de Brasilia, Pudahuel. F: 11 de agosto de 2016; OL: Ciclo de Guitarra Universidad de Chile, Concierto de Temporada "Fiesta Latinoamericana", Teatro Municipal de San Joaquín. F: 5 de septiembre de 2016; OL: Ciclo Lunes Musicales, "Música chilena y sudamericana", Sala América, Biblioteca Nacional. F: 8 de septiembre de 2016; OL: Dirección de Extensión, Comunicaciones y Relaciones Universitarias (DECRU), en conjunto con la Facultad de Humanidades y Educación de la Universidad de Atacama, Concierto para guitarra, Biblioteca del Liceo José Antonio Carvajal, Copiapó. F: 11 de septiembre de 2016; OL: Ciclo de Conciertos de la Liga Chileno-Alemana, Concierto de Guitarra "Ecos Latinoamericanos"; Int: Ximena Matamoros (guitarra). 
Matamoros Figueroa, Ximena (arr. Elías Allendes). TM: Océano (Blues) (2001) para violín y piano; F: 1 de agosto de 2016; OL: Panorama Musical Chileno $\mathrm{N}^{\mathrm{o}} 4$, Concierto para violín y piano, Sala América de la Biblioteca Nacional; Int: Elías Allendes Osses (violín), Patricia Castro Ahumada (piano). Versión para guitarra; F: 5 de septiembre de 2016; OL: Ciclo Lunes Musicales, "Música Chilena y Sudamericana", Sala América, Biblioteca Nacional. F: 8 de septiembre de 2016; OL: Dirección de Extensión, Comunicaciones y Relaciones Universitarias (DECRU), en conjunto con la Facultad de Humanidades y Educación de la Universidad de Atacama, Concierto para guitarra, Biblioteca del Liceo José Antonio Carvajal, Copiapó. F: 11 de septiembre de 2016; OL: Ciclo de Conciertos de la Liga Chileno-Alemana, Concierto de Guitarra "Ecos Latinoamericanos"; Int: Ximena Matamoros (guitarra).

TM: *Balada de la montaña (2013) para guitarra; F: 11 de agosto de 2016; OL: Ciclo de Guitarra Universidad de Chile, Concierto de Temporada "Fiesta Latinoamericana”, Teatro Municipal de San Joaquín. F: 5 de septiembre de 2016; OL: Ciclo Lunes Musicales, "Música Chilena y Sudamericana", Sala América, Biblioteca Nacional. F: 8 de septiembre de 2016; OL: Dirección de Extensión, Comunicaciones y Relaciones Universitarias (DECRU), en conjunto con la Facultad de Humanidades y Educación de las Universidad de Atacama, Concierto para guitarra, Biblioteca del Liceo José Antonio Carvajal, Copiapó. F: 8 de septiembre de 2016; OL: Ceremonia Universidad de Atacama, Teatro del Centro Cultural de Copiapó. F: 11 de septiembre de 2016; OL: Ciclo de Conciertos de la Liga Chileno-Alemana, Concierto de Guitarra "Ecos Latinoamericanos"; Int: Ximena Matamoros (guitarra).

Maupoint Álvarez, Andrés. TM: El iluminado (2002) para piano; F: 6 de julio de 2016; OL: Temporada Oficial Departamento de Música y Sonología, Facultad de Artes de la Universidad de Chile, Ciclo Siglos XIX y XX, Música instrumental contemporánea, Sala Isidora Zegers; Int: Andrés Maupoint (piano).

TM: Erste Kommentar zu "Le Tombeau d' une étoile" (2016) para guitarra; F: 24 de julio de 2016; OL: Ciclo de Guitarra, Referentes y estrenos, Departamento de Música y Sonología, Facultad de Artes de la Universidad de Chile, Museo Nacional de Bellas Artes; Int: Luis Orlandini Robert (guitarra).

TM: Veintiún miniaturas y una pieza final (2015) para flauta, fagot, viola, violonchelo, contrabajo y piano; F: 21 de septiembre de 2016; OL: Temporada Oficial de Conciertos 2016, Departamento de Música Sonología, Facultad de Artes de la Universidad de Chile, Sala Isidora Zegers; Int: DMUS ENSEMBLE (ensemble de profesores), Wilson Padilla Véliz (flauta), Jorge Espinoza (fagot), Felipe Marín (viola), María Fernanda Guerra (violonchelo), Lautaro Miranda (contrabajo), Andrés Maupoint (piano, director).

Mezzano, Cristián. TM: Londres 38 (49) (2016) para trombón, electrónica y proyección de imágenes; F: 22 de junio de 2016; OL: Proyecto "Desde el Centro 2", Taller $\mathrm{N}^{\circ}$ 8, Centro Cultural de Los Andes; Int: Cristián Mezzano (trombón), Francisco Silva (director del proyecto). 
Molina, Sebastián. TM: Destrucciones y construcciones (2015) para orquesta, obra que obtuvo el segundo lugar en el Concurso de Composición Luis Advis 2015; F: 9 de junio de 2016; OL: 4to. Concierto de Extensión de la Temporada Oficial, Choapa, Limarí, Elqui. F: 10 de junio de 2016; OL: 4to. Concierto, Temporada Oficial, Teatro Municipal de La Serena; Int: Orquesta Sinfónica de La Serena, Pablo Carrasco (director).

Mora López, Mario. TM: Calma (2007) para piano y electrónica; F: 25 de mayo de 2016; OL: Temporada Oficial del Departamento de Música y Sonología, Facultad de Artes de la Universidad de Chile, Gabinete de Electroacústica para la Música de Arte (GEMA), Sala Isidora Zegers; Int: Jorge Pepi-Alos (piano).

TM: Doo (2011) para flauta sola; F: 6 de julio de 2016; OL: Temporada Oficial Departamento de Música y Sonología, Facultad de Artes de la Universidad de Chile, Ciclo Siglos XIX y XX, Música instrumental contemporánea, Sala Isidora Zegers; Int: Wilson Padilla Véliz (flauta).

TM: Ephemeral (parte V-VI) (2012) para video, sonidos electrónicos, violonchelo y electrónica en tiempo real; F: 10 de agosto de 2016; OL: Temporada Oficial, Departamento de Música y Sonología, Facultad de Artes de la Universidad de Chile, Ciclo Siglos XX y XXI, Gema (Gabinete de Electroacústica para la Música de Arte), Sala Isidora Zegers; Int: Roberto Becerra (violonchelo).

TM: Nonática (2012), homenaje a Cirilo Vila para flauta, clarinete, violín, viola, violonchelo y piano; F: 21 de septiembre de 2016; OL: Temporada Oficial de Conciertos 2016, Departamento de Música y Sonología, Facultad de Artes de la Universidad de Chile, Sala Isidora Zegers; Int: DMUS ENSEMBLE (ensemble de profesores), Andrés Maupoint (director).

TM: Lucuática (2016), homenaje a Cirilo Vila para flauta, clarinete, violín, viola, violonchelo y piano; F: 21 de septiembre de 2016; OL: Temporada Oficial de Conciertos 2016, Departamento de Música y Sonología, Facultad de Artes de la Universidad de Chile, Sala Isidora Zegers; Int: DMUS ENSEMBLE (ensemble de profesores), Andrés Maupoint (director).

Moreira, Francisco. TM: Ay mamita (2013) para conjunto vocal e instrumental; F: 26 de abril de 2016; OL: Concierto gratuito, Librería Le Monde Diplomatique, Santiago; Int: Grupo Sexto Piso, Claudio Acevedo (director).

TM: Broches de candelitas (2015) para conjunto vocal e instrumental; F: 26 de abril de 2016; OL: Concierto gratuito, Librería Le Monde Diplomatique, Santiago; Int: Grupo Sexto Piso, Claudio Acevedo (director).

TM: Con tus manos con mis manos (2015) para conjunto vocal e instrumental; F: 26 de abril de 2016; OL: Concierto gratuito, Librería Le Monde Diplomatique, Santiago; Int: Grupo Sexto Piso, Claudio Acevedo (director).

Muñoz Arellano, Andrés. TM: Espacios que suenan. Performance sonora. Consiste en tres partituras independientes de tres compositores. Cada uno realiza una acción sonora en un piso del estacionamiento, y los asistentes los irán recorriendo en 
forma ascendente hasta llegar al Goethe Institut; F: 30 de julio de 2016; OL: VII Encuentro Internacional de Compositores, Instituto de Música, Facultad de Artes de la Pontificia Universidad Católica de Chile, Goethe Institut; Int: Pablo Aranda, Antonio Carvallo y Andrés Núñez. Ver además las entradas bajo este título de obras correspondientes a Pablo Aranda y Antonio Carvallo Pinto.

Mussorgsky, Modesto / Víctor Hugo Toro. TM: Cuadros de una exposición (versión original para piano, 1874) versión para orquesta de Víctor Hugo Toro; F: 3 de agosto de 2016; OL: Temporada de Conciertos 2016, Corporación Cultural de Las Condes; Int: Orquesta de Cámara de Chile, Víctor Hugo Toro (director).

Núñez, David. TM: *Lego II, escena sonora para instrumentos de juguete (2016); F: 31 de julio de 2016; OL: VII Encuentro Internacional de Compositores, "Música y escena", Instituto de Música, Facultad de Artes de la Pontificia Universidad Católica de Chile, Goethe Institut; Int: Ensamble de instrumentos de juguete: Andrés Núñez, Eduardo Sato, Fernanda Ortega, Mauricio Carrasco, Carolina Sagredo (puesta en escena).

Osorio González, Daniel. TM: Spiegelung (2013) para video y electroacústica; F: 10 de agosto de 2016; OL: Temporada Oficial, Departamento de Música y Sonología, Facultad de Artes de la Universidad de Chile, Ciclo Siglos XX y XXI, Gema (Gabinete de Electroacústica para la Música de Arte), Sala Isidora Zegers.

Parra Sandoval, Violeta. TM: Anticueca $\mathrm{N}^{\circ} 1$ (1960) para guitarra; F: 26 de abril de 2016; OL: Inauguración del Año Académico 2016 del Instituto de Chile, Auditorio del Instituto de Chile; Int: Luis Orlandini Robert (guitarra).

TM: Fantasía de Violeta Parra (arr. Nicolás Cortés Castillo) (2012), versión para quinteto de bronces; F: 26 de mayo de 2016; OL: Semana de la Educación, Concierto Educativo Bill Brass, Departamento de Musica y Sonología, Facultad de Artes de la Universidad de Chile, Sala Isidora Zegers; Int: Quinteto de Bronces Bill Brass: Wilson García (trompeta), Pablo Carreño (trompeta), Sebastián Lizana (corno francés), David Naranjo (trombón) y Nicolás Cortés (tuba), Claudio Merino (comentarios).

(arr. Guillermo Rifo). TM: La jardinera (1953), versión para orquesta; F: 9, 10, 15 y 16 de septiembre de 2016; OL: Concierto $\mathrm{N}^{\circ} 17$, Música Chilena, Temporada Oficial 2016 de la Orquesta Sinfónica de Chile (OSCH), Centro de Extensión Artística y Cultural de la Universidad de Chile, Teatro de la Universidad de Chile; Int: Orquesta Sinfónica de Chile, Guillermo Rifo (director).

(arr. Guillermo Rifo). TM: Gracias a la vida (1966), versión para orquesta; F: 9, 10, 15 y 16 de septiembre de 2016; OL: Concierto $\mathrm{N}^{\circ}$ 17, Música Chilena, Temporada Oficial 2016 de la Orquesta Sinfónica de Chile (OSCH), Centro de Extensión Artística y Cultural de la Universidad de Chile, Teatro de la Universidad de Chile; Int: Orquesta Sinfónica de Chile, Guillermo Rifo (director).

TM: Gracias a la vida (1966), improvisación para piano; $F: 17$ de agosto de 2016; OL: Ciclo de Grandes Pianistas, Teatro Municipal de Santiago; Int: Gabriela Montero (piano, Venezuela). 
(arr. Guillermo Rifo). TM: Run Run se fue pa'l norte (1966), versión para orquesta; F: 9, 10, 15 y 16 de septiembre de 2016; OL: Concierto $\mathrm{N}^{\circ} 17$, Música Chilena, Temporada Oficial 2016 de la Orquesta Sinfónica de Chile (OSCH), Centro de Extensión Artística y Cultural de la Universidad de Chile, Teatro de la Universidad de Chile; Int: Orquesta Sinfónica de Chile, Guillermo Rifo (director).

(arr. Guillermo Rifo). TM: Volver a los diecisiete (1966), versión para orquesta; F: 9, 10, 15 y 16 de septiembre de 2016; OL: Concierto $\mathrm{N}^{\circ} 17$, Música Chilena, Temporada Oficial 2016 de la Orquesta Sinfónica de Chile (OSCH), Centro de Extensión Artística y Cultural de la Universidad de Chile, Teatro de la Universidad de Chile; Int: Orquesta Sinfónica de Chile, Guillermo Rifo (director).

TM: El joven Sergio (1957); F: 15 de septiembre de 2016; OL: Concierto para guitarra, La Maison de l'Amérique Latine (Casa de América Latina), París, Francia; Int: César Farías Huenuqueo (guitarra).

Pepi Alos, Jorge. TM: La vida maravillosa y burlesca del café (1999) para orquesta; F: 31 de agosto de 2016; OL: Temporada Oficial Universidad de Santiago de Chile, Concierto "Cantatas humeantes como un café", Aula Magna de la Universidad de Santiago de Chile (USACH). F: 14 de septiembre de 2016; OL: Temporada Oficial, Universidad de Santiago de Chile, Centro Cultural Gabriela Mistral (GAM); Int: Orquesta Clásica Universidad de Santiago de Chile, Nicolas Rauss (director, Suiza).

Pérez, Delfina. TM: Armando el gondoliero (1858) para piano; F: 28 de septiembre de 2016; OL: Temporada Oficial, Departamento de Música y Sonología, Facultad de Artes de la Universidad de Chile, Ciclo de piano, Sala Isidora Zegers; Int: Kenya Godoy (piano).

Pinto D'Aguiar, Felipe. TM: Litoral (2010-2015) para consort de guitarras; F: 23 de junio de 2016; OL: Concierto de presentación CD CGCL-Micorriza del Consort Guitarrístico de Chile, Salón de Honor, Pontificia Universidad Católica de Valparaíso; Int: Consort Guitarrístico de Chile.

Quilapayún/ Nicolás Guillén (arr. Guillermo Rifo). TM: La muralla (1971), versión para orquesta; F: 9, 10, 15 y 16 de septiembre de 2016; OL: Concierto N ${ }^{\circ} 17$, Música Chilena, Temporada Oficial de la Orquesta Sinfónica de Chile (OSCH), Centro de Extensión Artística y Cultural de la Universidad de Chile, Teatro Universidad de Chile; Int: Orquesta Sinfónica de Chile, Guillermo Rifo (director).

Ramírez Ávila, Hernán. TM: Balada (2002) para soprano y piano, basada en un texto de Gabriela Mistral; F: 16 de junio de 2016; OL: Concierto de canto y piano, Academia Chilena de Bellas Artes, Salón de Honor del Instituto de Chile; Int: Camila García (soprano), Jacqueline Urízar (piano).

Rifo Suárez, Guillermo. TM: O+1 (2011) para oboe; F: 2 de mayo de 2016; OL:Presentación del disco Nuevos aires chilenos para oboe, Salón Mecesup, Departamento de Música de la Universidad de La Serena. F: 6 de mayo de 2016; OL: Presentación del disco Nuevos aires chilenos para oboe, Auditorio, Instituto de Chile. F: 9 de mayo de 2016; OL: Presentación del disco Nuevos aires chilenos para oboe, Teatro Caixa Preta, Universidad Federal de Santa María, Brasil; Int: José Luis Urquieta Plaza (oboe). 
Robles Gutiérrez, Manuel. TM: Primer Himno Nacional (1918) para voz y piano, texto de Bernardo Vera y Pintado; F: 29 de septiembre de 2016; OL: Ceremonia de celebración de los 52 años del Instituto de Chile, Salón de Honor del Instituto de Chile; Int: Francisco Huerta (tenor), Patricia Castro Ahumada (piano).

Rojas, Guillermo. TM: Divertimento (Moderato-Allegro) (1987) para fagot y piano, obra dedicada a Emilio Donatucci; F: 8 de agosto de 2016; OL: Temporada Oficial de Conciertos, Departamento de Música y Sonología, Facultad de Artes de la Universidad de Chile, Ciclo Música Chilena y Latinoamericana, Biblioteca Nacional; Int: Jorge Espinoza (fagot), Patricia Castro Ahumada (piano).

Romo Cartagena, René. TM: Ecos de un susurro distante (2015) para flauta y electrónica en tiempo real; F: 25 de mayo de 2016; OL: Temporada Oficial del Departamento de Música y Sonología, Facultad de Artes de la Universidad de Chile, Gabinete de Electroacústica para la Música de Arte (GEMA), Sala Isidora Zegers; Int: Richard Hazard (flauta).

Salinas Álvarez, Horacio. TM: Cristalino (1987); F: 15 de septiembre de 2016; OL: Concierto para guitarra, La Maison de l'Amérique Latine (Casa de América Latina), París, Francia; Int: César Farías Huenuqueo (guitarra).

Sánchez Dittborn, Juan Antonio. TM: Tonada por despedida (1999); F: 15 de septiembre de 2016; OL: Concierto para guitarra, La Maison de l'Amérique Latine (Casa de América Latina), París, Francia; Int: César Farías Huenuqueo (guitarra).

Sandoval, Felipe. TM: La otra mejilla (2015) para conjunto vocal e instrumental; F: 26 de abril de 2016; OL: Concierto gratuito, Librería Le Monde Diplomatique, Santiago; Int: Grupo Sexto Piso, Claudio Acevedo (director).

Santa Cruz Wilson, Domingo. TM: Tres piezas (1. Canción, lento y cadencioso como una tonada, 2. Recitativo-grave, 3. Arabesco-movido) (1936) para violín y piano; F: 1 de agosto de 2016; OL: Panorama Musical Chileno $\mathrm{N}^{\circ}$ 4, Concierto para violín y piano, Sala América de la Biblioteca Nacional; Int: Elías Allendes Osses (violín), Patricia Castro Ahumada (piano).

Sauvalle Vergara, Sergio (arr. Guillermo Rifo Suárez). TM: El corralero (1965), versión para orquesta; F: 9, 10, 15 y 16 de septiembre de 2016; OL: Concierto $\mathrm{N}^{\mathrm{o}} 17$, Música Chilena, Temporada Oficial 2016, Celebración Fiestas Patrias, Centro de Extensión Artística y Cultural de la Universidad de Chile, Teatro de la Universidad de Chile; Int: Orquesta Sinfónica de Chile, Guillermo Rifo (director).

Schidlowsky Gaete, León. TM: *Konstellation I (1969) para flauta, oboe y piano; F: 21 de julio de 2016; OL: Tercer concierto de El piano se toma la escena, $2^{\circ}$ Ciclo de piano y música de cámara, Concierto Homenaje a León Schidlowsky, Premio Nacional de Artes Musicales 2014, Goethe Institut y Centro Cultural Gabriela Mistral, Sala A1 del GAM; Int: Nicolás Faunes (flauta), Diego Villela (oboe), Fernanda Ortega (piano), Daniela Fugellie (comentarios).

TM: *Konstellation II (1971) para flauta, oboe y piano; $F: 21$ de julio de 2016; OL: Tercer concierto de El piano se toma la escena, $2^{\circ}$ Ciclo de piano 
y música de cámara, Concierto Homenaje a León Schidlowsky, Premio Nacional de Artes Musicales 2014, Goethe Institut y Centro Cultural Gabriela Mistral, Sala A1 del GAM; Int: Nicolás Faunes (flauta), Diego Villela (oboe), Fernanda Ortega (piano), Daniela Fugellie (comentarios).

TM: *Sound Poem (1977) para voz y piano; F: 21 de julio de 2016; $O L$ : Tercer concierto de El piano se toma la escena, $2^{\circ}$ Ciclo de piano y música de cámara, Concierto Homenaje a León Schidlowsky, Premio Nacional de Artes Musicales 2014, Goethe Institut y Centro Cultural Gabriela Mistral, Sala A1 del GAM; Int: Nancy Gómez (soprano), Fernanda Ortega (piano), Daniela Fugellie (comentarios).

TM: *Deutschland, ein Wintermärchen, $\mathrm{N}^{\circ} 5$ (Weltanschanschauug) (1980) para voz, flauta, oboe, piano, percusiones; F: 21 de julio de 2016; OL:Tercer concierto de El piano se toma la escena, $2^{\circ}$ Ciclo de piano y música de cámara, Concierto Homenaje a León Schidlowsky, Premio Nacional de Artes Musicales 2014, Goethe Institut y Centro Cultural Gabriela Mistral, Sala A1 del GAM; Int: Nancy Gómez (soprano), Nicolás Faunes (flauta), Diego Villela (oboe), Fernanda Ortega (piano), Diego Villela y Nicolás Faunes (percusión), Daniela Fugellie (comentarios ).

TM: *Gilgul (1980) para piano; F: 21 de julio de 2016; OL: Tercer concierto de El piano se toma la escena, $2^{\circ}$ Ciclo de piano y música de cámara, Concierto Homenaje a León Schidlowsky, Premio Nacional de Artes Musicales 2014, Goethe Institut y Centro Cultural Gabriela Mistral, Sala A1 del GAM; Int: Fernanda Ortega (piano), Daniela Fugellie (comentarios).

TM: *Partita (2002) para oboe; F: 21 de julio de 2016; OL: Tercer concierto de El piano se toma la escena, $2^{\circ}$ Ciclo de piano y música de cámara, Concierto Homenaje a León Schidlowsky, Premio Nacional de Artes Musicales 2014, Goethe Institut y Centro Cultural Gabriela Mistral, Sala A1 del GAM; Int: Diego Villela (oboe), Daniela Fugellie (comentarios).

TM: Farewell (2012) para flauta; F: 21 de julio de 2016; OL: Tercer concierto de El piano se toma la escena, $2^{\circ}$ Ciclo de piano y música de cámara, Concierto Homenaje a León Schidlowsky, Premio Nacional de Artes Musicales 2014, Goethe Institut y Centro Cultural Gabriela Mistral, Sala A1 del GAM; Int: Nicolás Faunes (flauta), Daniela Fugellie (comentarios).

Seves, Sebastián. TM: La abadesa (2011) para conjunto vocal e instrumental; F: 26 de abril de 2016; OL: Concierto gratuito, Librería Le Monde Diplomatique, Santiago; Int: Grupo Sexto Piso, Claudio Acevedo (director).

Silva Cárdenas, Francisco. TM: Lengua muerta (2015) para oboe; F: 2 de mayo de 2016; OL: Presentación del disco Nuevos aires chilenos para oboe, Salón Mecesup, Departamento de Música de la Universidad de La Serena. F: 6 de mayo de 2016; OL: Presentación del disco Nuevos aires chilenos para oboe, Auditorio, Instituto de Chile. F: 9 de mayo de 2016; OL: Presentación del disco Nuevos aires chilenos para oboe, Teatro Caixa Preta, Universidad Federal de Santa María, Brasil; Int: José Luis Urquieta Plaza (oboe). 
Silva Ponce, René. TM: "Espejismo II" (2015) para oboe; F: 2 de mayo de 2016; OL: Presentación del disco Nuevos aires chilenos para oboe, Salón Mecesup, Departamento de Música de la Universidad de La Serena. F: 6 de mayo de 2016; OL: Presentación del disco Nuevos aires chilenos para oboe, Auditorio, Instituto de Chile. F: 9 de mayo de 2016; OL: Presentación del disco Nuevos aires chilenos para oboe, Teatro Caixa Preta, Universidad Federal de Santa María, Brasil; Int: José Luis Urquieta Plaza (oboe).

Smith, Anna. TM: La sonrisa (siglo XIX) para piano; F: 28 de septiembre de 2016; OL: Temporada Oficial, Departamento de Música y Sonología, Facultad de Artes de la Universidad de Chile, Ciclo de piano, Sala Isidora Zegers; Int: Kenya Godoy (piano).

Solovera Roje, Aliocha. TM: Tramas discontinuas (2012); F: 21 de abril de 2016; OL: $2^{\circ}$ Concierto de Extensión de la Temporada Oficial, Choapa, Limarí, Elqui. F: 22 de abril de 2016; OL: $2^{\circ}$ Concierto Temporada Oficial, Teatro Municipal de La Serena; Int: Orquesta Sinfónica de La Serena, Francisco Núñez (director).

Soro Barriga, Cristina. TM: Vals brillante (siglo XIX) para piano; F: 28 de septiembre de 2016; OL: Temporada Oficial, Departamento de Música y Sonología, Facultad de Artes de la Universidad de Chile, Ciclo de piano, Sala Isidora Zegers; Int: Kenya Godoy (piano).

Soro Barriga, Enrique. TM: Storia d'una bimba (1900) para voz y piano; F: 15 de abril de 2016; OL: Examen de Canto Superior (III), Cátedra profesora Carmen Luisa Letelier; Departamento de Música y Sonología, Facultad de Artes de la Universidad de Chile, Sala Isidora Zegers; Int: Francisco Huerta (tenor), Alfredo Saavedra (piano). F: 1 de septiembre de 2016; OL: Concierto de Música Vocal de Chile y Latinoamérica, Academia Chilena de Bellas Artes, Instituto de Chile; Int: Carolina Muñoz (soprano), Patricia Castro Ahumada (piano).

TM: In souvenir (1901) para voz y piano; $F$ : 15 de abril de 2016; OL: Examen de Canto Superior (III), Cátedra profesora Carmen Luisa Letelier; Departamento de Música y Sonología, Facultad de Artes de la Universidad de Chile, Sala Isidora Zegers; Int: Francisco Huerta (tenor), Alfredo Saavedra (piano).

TM: Andante appassionato (1899-) para orquesta; F: 21 de abril de 2016; OL: Concierto homenaje que brindó la Fundación de Orquestas Juveniles e Infantiles (FOJI) al ex Presidente Patricio Aylwin, Catedral de Santiago; Int: Orquesta Sinfónica Nacional Juvenil, Rodolfo Fischer (director). F: 28 de julio de 2016; OL: Tercer Concierto Sinfónico de la Orquesta Sinfónica de Concepción, Teatro de la Universidad de Concepción; Int: Orquesta Sinfónica de Concepción, David Navarro Turres (director, residente en Bélgica).

TM: Trois petits morceaux (1900) para piano, obra dedicada al senador Ramón Ricardo Rozas; F: 26 de septiembre de 2016; OL: Concierto Enrique Soro, obras para piano, Sala América, Biblioteca Nacional; Int: María Blanca Mastrantonio (piano).

TM: Danza d'amore (Album per pianoforte) (1904), para piano, obra dedicada a don Pedro Montt Montt; F: 26 de septiembre de 2016; OL: Concierto 
Enrique Soro, obras para piano, Sala América, Biblioteca Nacional; Int: María Blanca Mastrantonio (piano).

TM: Morceau Lyrique. Nocturno $\mathrm{N}^{\mathrm{o}} 1$ (1904) manuscrito para piano; F: 26 de septiembre de 2016; OL: Concierto Enrique Soro, obras para piano, Sala América, Biblioteca Nacional; Int: María Blanca Mastrantonio (piano).

TM: Nocturno en do maggiore (Album per pianoforte) (1905), obra dedicada a don José Tocornal; F: 26 de septiembre de 2016; OL: Concierto Enrique Soro, obras para piano, Sala América, Biblioteca Nacional; Int: María Blanca Mastrantonio (piano).

TM: Recuerdos de Concepción, suite romántica (1922) para piano; F: 26 de septiembre de 2016; OL: Concierto Enrique Soro, obras para piano, Sala América, Biblioteca Nacional; Int: María Blanca Mastrantonio (piano).

TM: Vals lírico (1940) manuscrito para piano; F: 26 de septiembre de 2016; OL: Concierto Enrique Soro, obras para piano, Sala América, Biblioteca Nacional; Int: María Blanca Mastrantonio (piano).

TM: Elegía (1944) para piano, obra que conmemora la muerte de su esposa Adriana; F: 26 de septiembre de 2016; OL: Concierto Enrique Soro, obras para piano, Sala América, Biblioteca Nacional; Int: María Blanca Mastrantonio (piano).

TM: Preludio elegíaco $\mathrm{N}^{\circ} 1$ (1949) para piano, obra dedicada a Rosita Renard; F: 26 de septiembre de 2016; OL: Concierto Enrique Soro, obras para piano, Sala América, Biblioteca Nacional; Int: María Blanca Mastrantonio (piano).

Tradicional chileno. TM: Suite a la chilena, versión para quinteto de bronces; F: 26 de mayo de 2016; OL: Semana de la Educación, Concierto Educativo Bill Brass, Departamento de Música y Sonología, Facultad de Artes de la Universidad de Chile, Sala Isidora Zegers; Int: Quinteto de Bronces Bill Brass: Wilson García (trompeta), Pablo Carreño (trompeta), Sebastián Lizana (corno francés), David Naranjo (trombón) y Nicolás Cortés (tuba), Claudio Merino (comentarios).

Urrutia Blondel, Jorge. TM: Planté una mata de rosa (de Canciones y Danzas Campesinas de Chile, Cuaderno $\mathrm{N}^{\mathrm{o}}$ 2) (1936) para voz y piano; $F: 1$ de septiembre de 2016; OL: Concierto de Música Vocal de Chile y Latinoamérica, Academia Chilena de Bellas Artes, Instituto de Chile; Int: Carolina Muñoz (soprano), Patricia Castro Ahumada (piano). F: 29 de septiembre de 2016; OL: Ceremonia de celebración de los 52 años del Instituto de Chile, Salón de Honor del Instituto de Chile; Int: Francisco Huerta (tenor), Patricia Castro Ahumada (piano).

TM: Vos sois la estrella más linda (de Canciones y Danzas Campesinas de Chile, Cuaderno $\mathrm{N}^{\circ}$ 2) (1936) para voz y piano; F: 29 de septiembre de 2016; OL: Ceremonia de celebración de los 52 años del Instituto de Chile, Salón de Honor del Instituto de Chile; Int: Francisco Huerta (tenor), Patricia Castro Ahumada (piano).

Vera Rivera, Santiago. TM: Suite Violeta (1994) para piano; F: 8 de junio de 2016; OL: Temporada Oficial Departamento de Música y Sonología, Facultad de Artes de 
la Universidad de Chile, Ciclo de piano, Uniendo naciones desde el piano. Piano a cuatro manos, Sala Isidora Zegers; Int: Svetlana Kotova y Rika Uchida (piano).

TM: *Orogénika II, Cristales de los Andes (2016) para violín y orquesta; F: 24 de agosto de 2016; OL: Temporada de Conciertos Universidad de los Andes, Aula Magna de la Universidad de los Andes; Int: Dorian Lamotte (violín), Camerata Universidad de los Andes, Eduardo Browne (director).

Vila Castro, Cirilo. TM: Secuencia. Momentos para cuarteto de cuerdas (1. Lento muy expresivo, 2. Movido agitato, 3. Lento, 4. Movido violento, 5. Lento, 6. Movido-con ferocidad, 7 Lento-desolado) (1964); F: 20 de julio de 2016; OL: "El corazón piensa", Homenaje al Maestro Cirilo Vila, Sala Isidora Zegers, Facultad de Artes de la Universidad de Chile; Int: Compañía de Música Contemporánea: Dustin Cassonet (violín), Héctor Canales (violín), Fabián Esparza (viola), Sebastián Mercado (violonchelo), Carlos Valenzuela Ramos (director).

TM: Poemas (1965) para piano; F: 20 de julio de 2016; OL: "El corazón piensa", Homenaje al Maestro Cirilo Vila, Sala Isidora Zegers, Facultad de Artes de la Universidad de Chile; Int: Compañía de Música Contemporánea: Julio Torres (piano), Carlos Valenzuela Ramos (director).

TM: Canto (1968) para ensamble; F: 20 de julio de 2016; OL: "El corazón piensa”, Homenaje al Maestro Cirilo Vila, Sala Isidora Zegers, Facultad de Artes de la Universidad de Chile; Int: Compañía de Música Contemporánea. Carlos Valenzuela Ramos (director).

TM: Invocación (1985) para violín y piano; F: 20 de julio de 2016; OL: "El corazón piensa”, Homenaje al Maestro Cirilo Vila, Sala Isidora Zegers, Facultad de Artes de la Universidad de Chile; Int: Compañía de Música Contemporánea: Dustin Cassonet (violín), Julio Ramos (piano), Carlos Valenzuela Ramos (director).

TM: Del diario de viaje de Johann Sebastian. Pequeño vals a la luz de la luna o Una invitación al vals con luna de Arnold Schoenberg (2001) para violonchelo y piano; F: 20 de julio de 2016; OL: "El corazón piensa", Homenaje al Maestro Cirilo Vila, Sala Isidora Zegers, Facultad de Artes de la Universidad de Chile; Int: Compañía de Música Contemporánea: Sebastián Mercado (violonchelo), Julio Torres (piano), Carlos Valenzuela Ramos (director).

TM: De sueños y evanescencias (1. Sisífo, 2. Acción de gracia, 3. Copla, 4. Canción de amor, 5. Parábola) (2003) para mezzosoprano, flauta, violonchelo y piano; F: 20 de julio de 2016; OL: "El corazón piensa", Homenaje al Maestro Cirilo Vila, Sala Isidora Zegers, Facultad de Artes de la Universidad de Chile; Int: Compañía de Música Contemporánea: Claudia Godoy (mezzosoprano), Roberto Cisternas V. (flauta), Sebastián Mercado (violonchelo), Julio Torres (piano), Carlos Valenzuela Ramos (piano y dirección).

TM: Lento estático y evocativo (1985) para violín y piano; $F$ : 1 de agosto de 2016; OL: Panorama Musical Chileno No 4, Concierto para violín y piano, Sala América de la Biblioteca Nacional; Int: Elías Allendes Osses (violín), Patricia Castro Ahumada (piano). 
TM: Rapsodia chilensis (Lento sostenuto) (1986) para piano; F: 8 de agosto de 2016; OL: Temporada Oficial de Conciertos, Departamento de Música y Sonología, Facultad de Artes de la Universidad de Chile, Ciclo Música Chilena y Latinoamericana, Biblioteca Nacional; Int: Patricia Castro Ahumada (piano).

Zamora Pérez, Carlos. TM: Vilama - La muerte del río (2014) para orquesta; F: 15 y 16 de abril de 2016: OL: Temporada Oficial 2016, Concierto $\mathrm{N}^{\circ} 4$, Centro de Extensión Artística y Cultural de la Universidad de Chile, Teatro de la Universidad de Chile; Int: Orquesta Sinfónica de Chile, Alejandra Urrutia (directora).

Zegers Montenegro, Isidora. TM: El Califa de Bagdad (ca. 1823) para piano; F: 4 de mayo de 2016; OL: Lanzamiento del libro Descubriendo las partituras de Isidora Zegers, Departamento de Música y Sonología de la Facultad de Artes de la Universidad de Chile, Sala Isidora Zegers; Int: Patricia Castro Ahumada (piano).

TM: La Capricieuse (ca. 1823) para piano; F: 4 de mayo de 2016; OL: Lanzamiento del libro Descubriendo las partituras de Isidora Zegers, Departamento de Música y Sonología de la Facultad de Artes de la Universidad de Chile, Sala Isidora Zegers; Int: Patricia Castro y Cecilia Margaño (piano a cuatro manos).

TM: La Pomone (ca. 1823) para piano; F: 4 de mayo de 2016; OL: Lanzamiento del libro Descubriendo las partituras de Isidora Zegers, Departamento de Música y Sonología de la Facultad de Artes de la Universidad de Chile, Sala Isidora Zegers; Int: Cecilia Margaño (piano).

TM: Valze par Massimino (ca. 1823) para piano; F: 4 de mayo de 2016; OL: Lanzamiento del libro Descubriendo las partituras de Isidora Zegers, Departamento de Música y Sonología de la Facultad de Artes de la Universidad de Chile, Sala Isidora Zegers; Int: Patricia Castro Ahumada (piano). 